\title{
Web Service Negotiation Model Based on Cooperative Game Theory
}

\author{
Yanping Chen ${ }^{1}$, Wei Shi ${ }^{2}$, Jue Wang ${ }^{3}$,Huifen Yan $^{1}$,Chao Guo ${ }^{1}$ \\ ${ }^{1}$ School of Computer Science, Xi'an University of Posts and \\ Telecommunications, Xi'an, Shaanxi, China, 710121 \\ ${ }^{2} X i$ 'an Jiaotong University City College, Xi'an, Shaanxi, China, 710018 \\ ${ }^{3}$ School of Finance and Economics, Xi'an Jiaotong University, Xi'an, \\ Shaanxi, China, 710061 \\ chenyanping@xupt.edu.cn,sw8864@hotmail.com,wang2jue2@126.com \\ yanelise@163.com,guochao19920408@qq.com
}

\begin{abstract}
In the service composition life cycle, the target between service requester and service provider is quite different, which will cause the Web service SLA negotiation problem. The implementation of Web service relies on the interaction between the service provider and the service requester. In this article, the game model of Web Service negotiation process is proposed and solutions of the multi-participant and dynamic game model are gave. Then, we obtained the effective method of SLA generation. Experiments show that our method will ensure the common interests both service requesters and service providers.
\end{abstract}

Keywords: Web Service Negotiation, Cooperative Game Theory

\section{Introduction}

With the improvement of Internet technology, the Internet software has become more and more professional and modular, which lead to the rise of Web services. Web services are independent, modular applications which could be implemented by interactions between service providers and service requesters. As to service provider, it publishes services with specific descriptions; as to the requester of Web service, it can access and invoke services through the network. These two roles (Service Provider and Service Requestor) are both necessary in the generation process of service level agreements (SLA) [1].

Web services implementation process can be represented by the game theory, in which the service requestor could choose whether to accept services after service providers published services into service registry. Service requesters tend to reduce the price and increase the quality of a Web service while service providers are quite opposite. This difference leads to the Web service negotiation problem. The negotiation goal of Web services is to maximize interests of all participants, by negotiation, Web services will execute quickly and efficiently. The target of Web service negotiations is SLA: a bargaining agreement between requestors and providers, which should ensure the common interests of both sides.

Relative researches could be divided to two sorts: one-to-one static Web Service negotiation[2-9] and complex negotiation[10-17]. However, all of these studies considered one-to-one static negotiation process while the SLA automatic generation algorithm is not achieved. In this article, we will study services negotiation process by game theory. By this means, the behavior of the participants could be depicted, and different types of equilibrium can be achieved to automaticlly generate SLA. 
Firstly, this article proposed the game model of Web Service negotiation process. Then, we describe the dynamic foundation of SLA and solute the multi-participant and dynamic game model. Furthermore, we draws the effective method of SLA generation. Finally, experimental results show that our method will ensure the common interests of service requesters and service providers.

\section{2 .Web Services Negotiation Framework}

In the Internet, there exists such services that have same functions but differ in non-functions. QoS(Quality of Service) values of Web services are the combining of non-functional properties, which is the basis on service selection of Web service requesters ${ }^{[18]}$. According to the impact on the utilities of service requesters, QoS attributes are divided into two categories: negative effect attributes $P_{i}$ and positive effect attributes $Q_{j}$.

Negative effect attributes means service requester expect a lower value, however, service providers have oposite interest goal. That is, $P_{i}$ is negatively correlated with the requestor's objective function and positively correlated with the provider's objective function. For example, as to price, requesters want to cut it while providers prefer to increase it.

Positive effect attributes means service requesters tend to increase it while service providers tend to decrease it to reduce the costs of services. That is, $Q_{j}$ is positively correlated with the requestor's objective function and negatively correlated with the provider's objective function.

According to the above, the goal of Web Service negotiation is get a SLA which can satisfy the need of service provider and service requestor. Obviously, SLA involved in both provider and requestor, thus, there is a game relationship between them. Game theory is the effective tools for confliction in multi-agent Specifically, the negotiation process of Web Service SLA is as following:

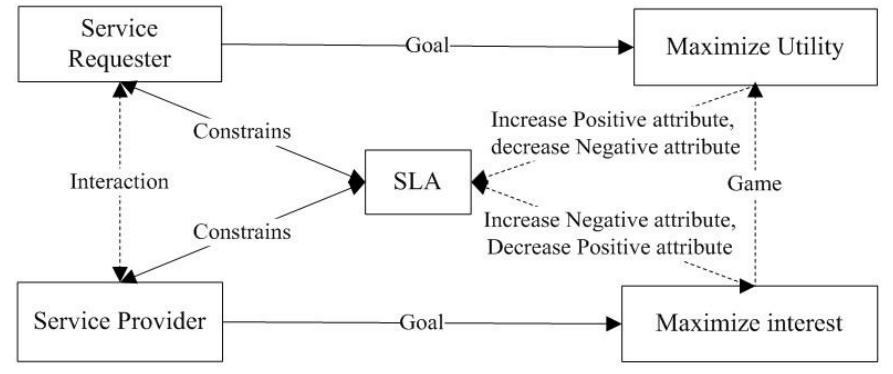

Figure 1. Game Relationship of Web Service Negotiation

In Figure 1, the key problem is to determine the QoS attributes in Web Service negation process, and the determined QoS value should satisfy both negotiation sides. How to get the determined QoS is also the core of this paper. Let's firstly give some basic definitions.

Definition 1. SLA is the agreement generated by all participants of negotiation, which determines the QoS level of services. A SLA can be expressed as a triple $(H, S, R)$ :

- $H=\left\{H_{r}, H_{p}\right\}$ is a set that contains two players: service provider $H_{p}$ and service requester $H_{r}$.

- $S=\left\{s_{1}, s_{2}, \ldots, s_{n}\right\}$ is a set of the service attributes, where $s_{i}(i=1,2, \ldots, k)$ refers to its functional attributes, $s_{j}(j=k+1, k=2, \ldots, n)$ refers to its non-functional attributes. Because that the functional properties of all the services are constant, we only consider non-functional properties $s_{k}, s_{k+1}, \ldots, s_{n}$, in this paper can be further 
expressed as, $S=\left\{P_{1}, P_{2}, \ldots, P_{n}, Q_{1}, Q_{2}, \ldots, \mathrm{Q}_{\mathrm{N}}\right\}$.

- $R=\left(R_{r}, R_{p}\right)$ refers to the Responsibilities.

As a constraint to negotiation process, SLA must ensure that the objective functions of all sides are optimal. This principle mainly reflects the stability and the fairness of SLA. The stability implies that the QoS levels required by SLA are acceptable to both parties, which means both sides get a improvement which made them not to default through negotiation. Fairness requires the equal treatment of both sides. It is easier to accept a fair deal or cooperation programs. But if people think that a solution is unfair, they often refuse to accept them. So fairness is related to the long-term stability. We believe that in order to actually work in the short term and long term, SLA must consider the stability and fairness at the same time.

\section{Game Analysis of SLA Negotiation Process}

\subsection{Introduction to Game Theory}

The Game Theory is the "theory about games". It refers to that some individuals or groups which faced certain environmental conditions and rules, simultaneously or successively select strategy obtain the results ${ }^{[19]}$. Here is the game's standard definition:

Definition 2. A game is a process that the participants select strategies from their set according to some rules and get the game results ${ }^{[19]}$. It can be represented by a triple $\operatorname{array}(H, S, \Pi)$.

Here,

$H=\left\{H_{1}, H_{2}, \ldots, H_{n}\right\}$ is the set of participants of Web service negotiation. In this article the participants of the game are Web service requestors and providers. Thus, the sets can be rewritten as $H=\left\{H_{r_{1}}, H_{r_{2}}, \ldots, H_{r_{n}}, H_{p_{1}}, H_{p_{2}}, \ldots, H_{p_{n}}\right\}$.

$s=S_{1} * S_{2} * \ldots * S_{n}$ is the set of strategies. A strategy set is a vector $S_{i}=\left(s_{1}, s_{2}, \ldots, s_{n}\right)$ and $S_{j}$ is a strategy of $S_{i}$. In this article, the strategies is the selection of QoS. Thus, the sets can be rewritten as $S_{i}=\left(P_{1}, P_{2}, \ldots, P_{n}, Q_{1}, Q_{2}, \ldots, Q_{N}\right)$, or $S=(P, Q)$.

$\Pi=\left(\pi_{1}, \pi_{2}, \ldots, \pi_{n}\right)$ is the set of payment function. $\pi_{i}: \mathrm{s} \rightarrow \mathrm{R}$ is the objective function of a participant. In this article, $\pi_{r}=\pi_{r_{0}}-P+\beta Q, \pi_{p},=P-\left(c_{0}+\gamma Q\right)$.

Since the objective of this study was to obtain SLA formation mechanism, we need to consider cooperative game analysis. Cooperative game allows the existence of a formal cooperation agreement among participants. When the participants of cooperative game are more than two, the problem of "Alliance" may appear. So we need the analysis of Alliance Game Theory in that situation.

In cooperative game, because the behaviors are determined by coordinated agreement, the individual strategy does not directly determine the outcome. So the most important factor is not the individual strategies of each player, but the 'distribution' of all the players' interests: $\theta=\left\{\theta_{1}, \theta_{2}, \ldots, \theta_{n}\right\}$. In this article, the distribution refers to the $\theta=\left\{\pi_{1}, \pi_{2}, \ldots, \pi_{n}\right\}$ which is determined by $\{P, Q\}$.

Meanwhile, because the subjective evaluation of the results will effect the final choice, we need to consider the utility configuration: $U=\left\{u_{1}, u_{2}, \ldots, u_{n}\right\}$, among which $u_{i}=u\left(\pi_{i}\right)$. Under the risk-neutral case $U=\theta$. Then the utility of both sides is equal to $\pi_{r}$ and $\pi^{p}$.

Finally, all the participants have their own "bottom line" in negotiations, which is also called break point: $D=\left\{d_{1}, d_{2}, \ldots, d_{n}\right\}$. This variable represents the distribution of the game when the negotiation is broke down, which is, the opportunity cost of negotiations. Generally, $d_{i}=0$. 
By the Pareto efficiency axiom, we can get the cooperative games Pareto border (Stability principle). Then based on the principle of fairness, we can get the solution of the game. On the other hand, integrated approach by Nash could solute the cooperative game faster.

\subsection{Game Equilibrium}

Based on the different requirements of results and different game settings, there are ways to get the game equilibrium.

(1) Fair solution

A fair solution must satisfy the symmetry condition: For a cooperative game $\{H, S, \theta, D\}$, only if $\left(\pi_{i}-d_{i}\right)=\left(\pi_{j}-d_{j}\right), i \neq j$, distribution $\theta^{*}$ is the solution of game $^{[19]}$.

Based on the symmetry condition, the method to achieve the solution is: firstly, choose the available distributions by Pareto efficiency axiom; secondly, find the optimal distribution by the symmetry condition.

(2) Nash solution

Nash method provides a simple solution for the problem of cooperative game, the distribution of "Nash product" obtained fairness and stability at the same time ${ }^{[19]}$.

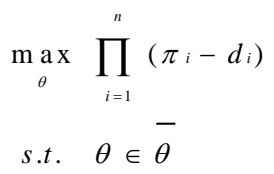

Easy to see that Nash solution analyzes the product of all the objective function $\prod^{n}\left(\pi_{i}-d_{i}\right)$, which shows the Nash solution are concerned about the fairness as well as effectiveness. On the one hand, Nash solution does not encourage individual rationality, on the other hand, it is also does not encourage absolutely fair.

\section{(3) Shapley Solution}

If the information is incomplete, the multi-game will inevitably lead to "Alliance" situation. In the alliance game which takes the above method into account, we can still get the solution. But the solution will easily be broken by the alliance of major participants ${ }^{[19]}$. So we need to consider the characteristic function of a game.

Characteristic function is defined as follows, for the alliance game of $n$ participants $A \in \Omega(N)$, no matter how the outside members of the alliance select strategy, members of the alliance can be guaranteed to achieve the greatest overall benefit $v(A)$. All $v(A)$ constitute the characteristic function of the alliance game.

To solve the alliance game, we need to define the dominance strategy (under the risk-neutral conditions), for the distributions $\theta$ and $\theta^{\prime}$ of a alliance game $B(H, v)$ and alliance A, if $\theta>\theta^{\prime}$ for any $i \in A$ were established and $\sum_{i \in A} \pi_{i} \leq v(\theta)$, we call that $\theta$ dominate $\theta^{\prime}$ about A. The set of solutions of the game is all the distributions $S^{*}$ that will not be dominated by other distributions $S$.

Because of the members of power set is often large, solute an alliance game is difficult, and here is another approach to solve it.

The optimal distribution of an alliance game should be the 'expected value' (Shapley value) of each participant. This value is a vector $\left(\varphi_{1}, \ldots, \varphi_{n}\right)$ : 
$\varphi_{i}=\sum_{A \in \Omega(N)} \frac{(n-k) !(k-1) !}{n !}[v(A)-v(A \backslash i)]$

Among them, $\mathrm{n}$ is the number of participants, $\mathrm{k}$ is the member population of $\mathrm{A}$. Shapley value $\left(\varphi_{1}, \ldots, \varphi_{n}\right)$ describes the expectation contribution of each participant, and it is the long-term stable solution of the alliance game while the 'powers' of all members is all used.

\subsection{Web Services Negotiation Game Model Based on SLA}

This article considers two main players of the game: service requestors $\left(H_{r^{r}}\right)$, service providers $\left(H_{p}\right)$, The payment functions of them are: $\pi_{r_{i}}=\pi_{r_{0}}-P+\beta Q$ and $\pi_{p i}=P-\left(c_{0}-\gamma Q\right)$. Among them, $\alpha, \beta, \gamma>0, P, Q$ is the selection of strategy. In the bargaining game, we believe $Q$ is fixed and $P$ is variable.

Obviously, $\theta=\left\{\pi_{r}, \pi_{p}\right\}, \beta>\gamma$, or all the costs of Web service will outweigh the benefits. Meanwhile, $\pi_{r_{0}}+\beta Q>c_{0}+\gamma Q$, or there will no Web service negotiation.

(1) One-to-one game model

In one-to-one game model, there are only one requestor and one provider. In this situation, based on the cooperative game theory, the two participants divide the common benefits: $C=\pi_{r}+\pi_{p}=\pi_{r_{0}}-c_{0}+(\beta-\gamma) Q$.

(2) Multi-participant game model

The service negotiation games with multiple service requesters or more service providers are known as multi-participant game model. There are three cases: 1 to $\mathrm{n}$ game, $\mathrm{n}$ to 1 game, $\mathrm{n}$ to $\mathrm{n}$ game. The key of the game is to 'carve up', which is to divide the benefits in the game after the SLA.

In this situation, because of exist three or more participants, some participants will unite to be alliance. For instance, there are a provider, a requester who cares about the service security and a requester cares about the transmission speed. If no alliance, the provider must consider both the service security and the transmission speed, and then pay a high cost. However, if the generation of SLA is consistent with the principle of a simple majority, the provider could cooperate with the requestor cares about the transmission speed and generate an alliance which tend to stipulate the SLA higher transmission speed and lower security, then all the members' benefits of the alliance are improved. Although the benefits of the requestor who cares about the service security damaged, his 'voice' in the SLA generation process is limited.

Since there may exist effective alliance in the multi-participant circumstance, we must use the alliance game method such as the Shapley solution to obtain the equilibrium.

\subsection{Game Equilibrium Analysis}

(1) One-to-one Game Equilibrium Analysis

In this situation, the total benefits of all the participants $C=\pi_{r}+\pi_{p}=\pi_{r o}-c_{0}+(\beta-\gamma) Q$ are fixed. So all the distributes is Pareto optimal.

We only consider the principle of fairness, which means:. ${ }^{\left(\pi_{r}-d_{r}\right)}=\left(\pi_{p}-d_{p}\right)$

$$
\begin{aligned}
& \pi_{r_{0}}-P+\beta Q-d_{r}=P-\left(c_{0}+\gamma Q\right)-d_{p} \\
& P^{*}=\frac{\pi_{r_{0}}+\beta Q+\left(c_{0}+\gamma Q\right)+d_{p}-d_{r}}{2}
\end{aligned}
$$

Where $P^{*}$ is the service price in the game equilibrium. 


$$
\text { Then } \pi_{r}=\frac{\pi_{r_{0}}+\beta Q-\left(c_{0}+\gamma Q\right)-d_{p}+d_{r}}{2}, \pi_{p}=\frac{\pi_{r_{0}}+\beta Q-\left(c_{0}+\gamma Q\right)+d_{p}-d_{r}}{2} \text {, }
$$

Because of the symmetry of the objective function, the Nash solution and fair solution should be no difference. Thus, we expand the Nash product under Pareto conditions:

$$
\begin{aligned}
& \left(\pi_{r}-d_{r}\right)\left(\pi_{p}-d_{p}\right) \\
= & \left(\pi_{r}-d_{r}\right)\left(C-\pi_{r}-d_{p}\right) \\
= & -\pi_{r}^{2}+\left(C+d_{r}-d_{p}\right) \pi_{r}+d_{p} d_{r}-C d_{r}
\end{aligned}
$$

The first order condition:

$$
\begin{aligned}
& \frac{\partial\left[-\pi_{r}{ }^{2}+\left(C+d_{r}-d_{p}\right) \pi_{r}+d_{p} d_{r}-C d_{r}\right]}{\partial P} \\
= & -\frac{\partial\left[-\pi_{r}^{2}+\left(C+d_{r}-d_{p}\right) \pi_{r}+d_{p} d_{r}-C d_{r}\right]}{\partial \pi_{r}} \\
= & 2 \pi_{r}-\left(C+d_{r}-d_{p}\right)
\end{aligned}
$$

Then, $\pi_{r}=\frac{C-d_{p}+d_{r}}{2}, \pi_{p}=\frac{C+d_{p}-d_{r}}{2}$.

Obviously, $\frac{\partial^{2}\left[-\pi_{r}{ }^{2}+\left(C+d_{r}-d_{p}\right) \pi_{r}+d_{p} d_{r}-C d_{r}\right]}{\partial P^{2}}=-2$.

The maximum value could be reached in $\pi_{r}=\frac{C-d_{p}+d_{r}}{2}, \pi_{p}=\frac{C+d_{p}-d_{r}}{2}$, so these are the solutions of the game. Then we can get that $P^{*}=\frac{\pi_{r_{0}}+\beta Q+\left(c_{0}+\gamma Q\right)+d_{p}-d_{r}}{2}$, which is consistent with the fair solution.

\section{(2) Multi-participant Game Equilibrium Analysis}

Since the multi-participant game situation involves "alliance", we need to consider the characteristic function of the game to solve it. Here are two kinds of principles to set the characteristic function: the principle of consensus and the principle of simple majority. To simplify the analysis, we consider the four participants, two service requestors and two service providers.

The set of feasible game distributions is:

$\left\{\left(\pi_{r_{1}}, \pi_{r_{2}}, \pi_{p_{1}}, \pi_{p_{2}}\right) \mid \pi_{i} \geq d_{i},\left(\pi_{r_{1}}-d_{r_{1}}\right)+\left(\pi_{r_{2}}-d_{r_{2}}\right)+\left(\pi_{p_{1}}-d_{p_{1}}\right)+\left(\pi_{p_{2}}-d_{p_{2}}\right)=C-D\right\} \quad$ Among them, $\mathrm{D}$ is the added benefits on the break points $d_{i}$. Notably, the net benefits $C-D$ at this time are divided into four parts. We need more QoS changes to meet the requirements instead of only P.

\section{A. The Principle of Consensus}

The principle of consensus means that the generation of SLA must consider all the participants' benefits. SLA generation process will break when one or more participants disagree. In this situation, there will no effective alliance. Now the characteristics function of alliance game are:

$v\left(\pi_{r_{1}}, \pi_{r_{2}}, \pi_{p_{1}}, \pi_{p}=\right) C-D, \quad v($ other cases $)=0$

According to the formula (1), the Shapley value of each participants are:

$\varphi_{r_{1}}=\varphi_{r_{2}}=\varphi_{p_{1}}=\varphi_{p_{2}}=\frac{(C-D)}{4}$

The game solution is: 


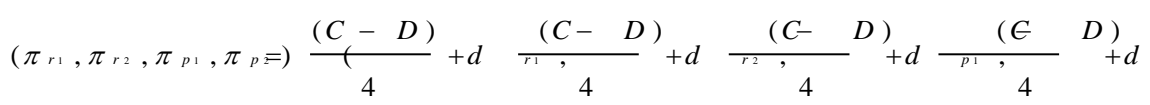

Obviously, to the participants have different ${ }^{d i}$, their actual benefits will differ. But when we consider the ${ }^{d_{i}}$ as endowments, the principle of consensus meets the symmetry condition and will lead to the fairer negotiation results. And because of $C>D$, these results will be stable in the long-term.

\section{B. The Principle of Simple Majority}

The principle of simple majority means that the generations of SLA need not to consider all the participants' benefits. SLA generation process will break when only if the major participants disagree.

In this situation, the alliance is effective.

$$
\begin{aligned}
& \left.v\left(\pi_{r_{1}}, \pi_{r_{2}}, \pi_{p_{1}}, \pi_{p} \equiv\right) v \quad\left(\pi_{r_{2}}, \pi_{p_{1}}, \pi \bar{F}_{2} v\right) \quad \pi_{r_{1}} \pi_{p_{1}} \#_{p_{p}} \nu\right) \quad \pi_{r_{1}} \pi \\
& =v\left(\pi_{r_{1}}, \pi_{r_{2}}, \pi_{p_{1}} \neq C-D\right.
\end{aligned}
$$

According to the formula (1), the Shapley value of each participant are (When $r_{2}, p_{1}, p_{2}$ united) :

$$
\varphi_{r 2}=\varphi_{p 1}=\varphi_{p}=\frac{(C-D)}{3}, \varphi_{r}=0
$$

The game solution is as follows:

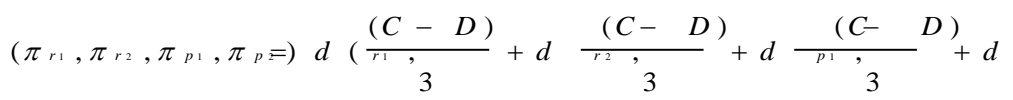

Obviously, this situation is bad to the participant not in the alliance. The result of the game has short-term stability but not has fairness. In the next step, $r_{1}$ could unit ${ }^{p_{1}}$ and by the new distribution $\left(\frac{(C-D)}{9}+d_{r_{1}}, \frac{4(C-D)}{9}+d_{p 1}, \frac{4(C-D)}{9}+d_{p 2}\right)$ and generate a new alliance. When the cost of default exists, this will lead to the non-optimal results.

\section{(3) Comparison of SLAs in different situations}

Based on the above analysis, in the case of one-to-one game, SLA is almost always equals to fair solution. In the multi-participant game, only the SLA under the principle of consensus has fairness.

To the participants with different $d_{i}$, their actual benefits will differ. $d_{i}$ is the distribution when the negotiation break down.

In the one-to-one game model, the fair solution is: $\pi_{r}=\frac{C-d_{p}+d_{r}}{2}, \pi_{p}=\frac{C+d_{p}-d_{r}}{2}$. When the requester is in a dominant position, $d_{r}>d_{p}$, then $\pi_{r}>\pi_{p}$. This means the dominant position will bring revenues, and vice versa.

In the 1 ton model, the fair solution is:

$\left(\pi_{r 1}, \pi_{r 2}, \pi_{p 1}, \pi_{p 2}\right)=\left(\frac{(C-D)}{4}+d_{r 1}, \frac{(C-D)}{4}+d_{r_{2}}, \frac{(C-D)}{4}+d_{p 1}, \frac{(C-D)}{4}+d_{p 2}\right)$, When

the requester is in a dominant position, $d_{r_{1}}>d_{r_{2}}, d_{p_{1}}, d_{p_{2}}$, then $\pi_{r_{1}}>\pi_{r_{2}}, \pi_{p_{1}}, \pi_{p_{2}}$. This means the dominant position will bring revenues, and vice versa.

The difference of market dominant position is determined by the different monopoly position, the monopolistic participant will be powerful in Web services negotiation. All the above discussions are based on complete information assumptions. However, participants may not know the true preferences of others. Then the participants can take some strategies to get close to it. 


\section{Simulations}

Based on the results of game analysis, this study will conduct simulation experiments under different conditions. We will use the random variables which comply with normal stdistribution to simulate the behaviors of game participants. Specifically, we will reflect rategy tendency by the mean of random variables and reflect the rational level by the variance changes.

\subsection{Design of Simulation Experiments}

(1) One-to-one game model

The fair solution and Nash solution is equivalent in one-to-one game model.

Because that there are many independent factors to the behaviors of game participants, we believe the benefits in one-to-one game model comply with normal distribution.

$$
\pi_{r} \sim N\left(\frac{C-d_{p}+d_{r}}{2}, \sigma_{r^{2}}{ }^{2}\right), \pi_{p} \sim N\left(\frac{C+d_{p}-d_{r}}{2}, \sigma_{p}{ }^{2}\right)
$$

Among them, $\sigma_{r}{ }^{2}, \sigma_{p}{ }^{2}$ represent the non-rational degree of requestors and providers. The higher rational degree will lead to the lower variance.

(2) Multi-participant game model

Because the case that under the principle of consensus is equal to the one-to-one situation, we just consider the principle of simple majority. At this part, we will use a Uniform distribution to determine the alliance generation when there are more than two potential partners, Such as:

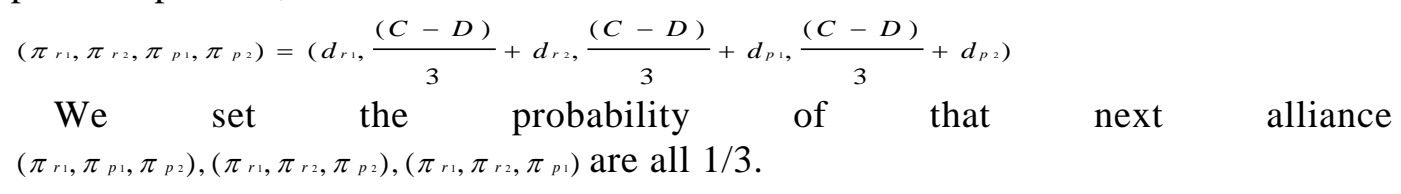

With the increase of the participants, the phenomenon of defaults may occur. Considering the default cost, we set that the one who change the QoS of SLA will pay the $\operatorname{costs} \varepsilon$.

\subsection{Parameter Settings}

(1) One-to-one game model

In this model, the equilibrium price is 1 . And we analyze the results in two cases: $d_{r}=d_{p}$ and $d_{r}>d_{p}$. Also, to analyze different market state, each case is divided into two distinct difference setting: rational $\left(\sigma_{r}{ }^{2}, \sigma_{p}{ }^{2}=0.05\right)$ and irrational $\left(\sigma_{r}{ }^{2}, \sigma_{p}{ }^{2}=0.3\right)$. The experimhent was divided into 20 groups, the no. 1 group negotiates 5 times, the no. 2 group negotiates 10 times, and so on. Finally, by comparing the average benefits of 20 groups, we analyze the difference between short-term results and long-term results and the factors of the results.This model uses pseudo-random number to simulate the behavior of participants in the game just little times. The participants outside the existing alliance will devote to design a higher benefits distribution which exclude the current highest benefit member and assigns the benefits to the members in the new alliance. If the 'highest member' is two or more, we use pseudo-random number to select the one outside the new alliance. 


\subsection{Comparison of Experiment Results}

(1) One-to-one game model

The market dominance is no difference:

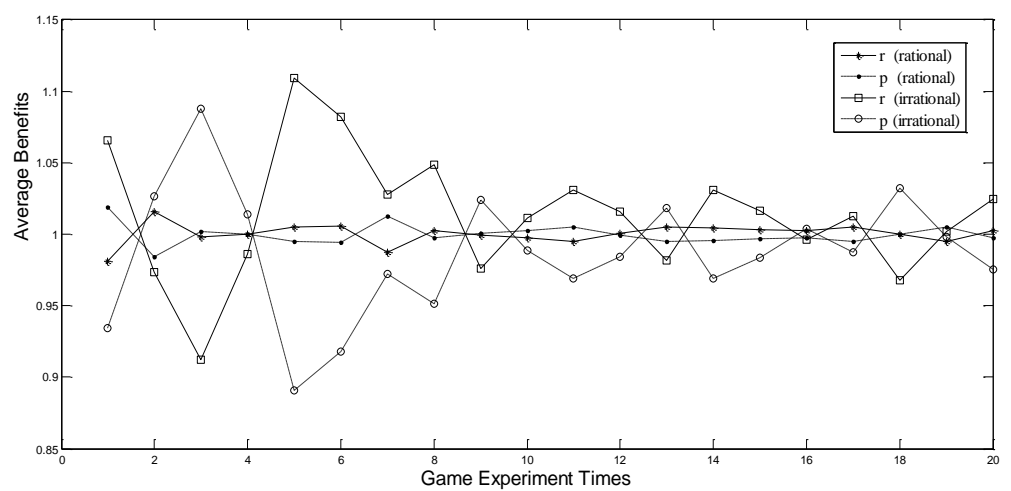

Figure 2. The Experiment Results when the Market Dominance is No Difference

Obviously, in this case, the more times people negotiate, the more stable that SLA results are and the fairer the long-term solutions are which is reflected in that the revenues of both participants are nearly equal. On the other hand, the rational and irrational situations significantly affect stability of significant SLA, a more rational negotiation process is expected to get a fair SLA program more quickly.

The market dominance has some differences:

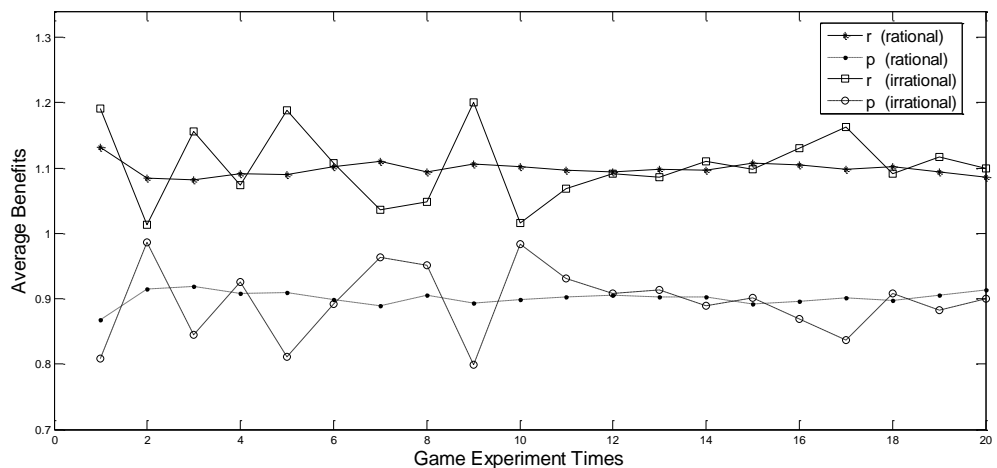

Figure 3. The Experiment Results when the Market Dominance has Differences

Similarly, in this case, the more times people negotiate, the more stable that SLA results are. The long-term solutions are not that fair because the market dominance has differences. But if we consider the net benefits, the results in this situation are still fair. On the other hand, the rational and irrational situations also significantly affect stability of significant SLA, a more rational negotiation process is expected to get a fair SLA program more quickly.

(2) Multi-participant game model

Considering that the alliance game only repeated 11 times (after period 11 the results cycled), we could obtain the proportions that each participant's share of the total revenue. 
Table 1. Results of Alliance Game

\begin{tabular}{cccccccccccccc}
\hline Periods & 1 & 2 & 3 & 4 & 5 & 6 & 7 & 8 & 9 & 10 & 11 & $\begin{array}{c}\text { Average } \\
\text { benefits }\end{array}$ \\
\hline $\mathrm{R} 1$ & 0.00 & 0.11 & 0.26 & 0.46 & 0.00 & 0.17 & 0.34 & 0.50 & 0.00 & 0.17 & 0.33 & 0.21 \\
\hline $\mathrm{R} 2$ & 0.33 & 0.00 & 0.15 & 0.35 & 0.50 & 0.00 & 0.17 & 0.34 & 0.50 & 0.00 & 0.17 & 0.23 \\
\hline $\mathrm{P} 1$ & 0.33 & 0.44 & 0.00 & 0.20 & 0.35 & 0.52 & 0.00 & 0.16 & 0.33 & 0.50 & 0.00 & 0.26 \\
\hline $\mathrm{P} 2$ & 0.33 & 0.44 & 0.59 & 0.00 & 0.15 & 0.32 & 0.49 & 0.00 & 0.17 & 0.33 & 0.50 & 0.30 \\
\hline & & & & & & & & & & & & & \\
\end{tabular}

Figure 4. Results of Alliance Game

It is easy to see that even though a single alliance game result is not fair, multi-period alliance game is consistent with the principle of fairness and has convergence trend. As is shown in table 1 , there is no obvious difference among each participant. Therefore, if possible, we should not take the principle of simple majority, however take the principle of consensus. But the principle of consensus may cause the low effectiveness in SLA generation.

\section{Conclusion and Future Works}

The purpose of this study is to solve the problems of Web service negotiation, namely the inefficiencies and default problems in SLA generation process. Therefore, the primary objective is to get an effective SLA generation mechanism that could ensure the respective benefits of Web service requesters and Web service providers, thereby reducing the SLA defaults. Specifically, this study yielded the following results.

On the basis of a simple Web service negotiation game equilibrium solution, we get the equilibrium solution in general Web service negotiation process which means the multi-participant game. Meanwhile the variables in game will not be limited to the price, but considering a variety of QoS attributes. This result reveals the methods to obtain a reliable and efficient SLA under different conditions. We used Matlab software simulation. By calibrating parameters, we get a stable game simulation framework and results.

Based on the game theory and simulation experiments, we got results which are consistent with our expectation. But because the limitation of time, we did not analyze the specific provisions of QoS attributes, and did not consider the other circumstances of Web service negotiation process. Therefore, we will study on these aspects in the next phase of work.

\section{Acknowledgments}

Supported by New Star Team of Xi'an University of Posts \& Telecommunications, Scientific Research Funded by Shaanxi Provincial Education Department (Program No.2013JK1130) and Xi'an science and technology plan projects (Program No.CXY1437 
$(8))$.

\section{References}

[1] IBM Corporation and Microsoft Corporation. UDDI Technical White Paper. 2000. http://uddi.xml.org/

[2] K Hashmi, E Najmi, Z Malik, A Rezgui. A Framework for Automated Service Negotiation. 2013 IEEE 6th International Conference on Service-Oriented Computing and Applications.pp.233-240, Dec. (2013).

[3] M Al-aaidroos, N Jailani, M Mukhtar. Agent-based Negotiation Framework for Web Service's SLA. 2011 7th International Conference on IT in Asia (CITA). pp.1-7, July. (2011).

[4] S Jiajia. Research on Game Theory Based Service Composition. Beijing: University of Science and Technology Beijing. (2011).

[5] Nguyen The Cuong, Niccolò De Caro, Federico Domínguez, Kris Steenhaut, Abdellah Touhafi. QoS Level Estimation for Web Service Composition in an Environmental Assessment System. 2013 19th IEEE Workshop on Local \& Metropolitan Area Networks. pp 1-3, April. (2013).

[6] X Zheng, P Martin, Kathryn Brohman.Applying Bargaining Game Theory to Web Services Negotiation.2010 IEEE International Conference on Services Computing. 218-225.

[7] D Pukhkaiev, T Kot, Larysa Globa, Alexander Schill. A Novel SLA-Aware Approach for Web Service Composition. EuroCon 2013. pp. 327-334, July. (2013).

[8] L Zhongjun, Z Hua, G Jing, YFangchun, Lin Rongheng, Multi-QoS effective prediction in web service selection, Web Technologies and Applications - 15th Asia-Pacific Web Conference, pp. 176-183, April (2013).

[9] L Shu-Lei, L Yun-Xiang, Z Fan, T Gui-Fen, J Ning, A dynamic Web services selection algorithm with QoS global optimal in Web services composition, Journal of Software, Vol. 18, No. 3, pp. 646-656, Mar. (2007).

[10] F SIALA, K GHEDIRA. A Multi-Agent Selection of Web Service Providers Driven by Composite QoS. The Sixteenth IEEE Symposium on Computers and Communications. pp. 55-66, June. (2011).

[11] M Rathore, U Suman. An ARSM Approach using PCB-QoS Classification for Web Services: A Multi-Perspective View. Proceedings of the 2013 International Conference on Advances in Computing, Communications and Informatics. pp. 165-171, Aug. 2013.

[12] M Abundo, V Di Valerio, V Cardellini, F Lo Presti. Bidding Strategies in QoS-Aware Cloud Systems Based on N-Armed Bandit Problems. 2014 IEEE 3rd Symposium on Network Cloud Computing and Applications. pp. 38-45, Feb.( 2014).

[13] K Hashmi, A Alhosban, E Najmi and Z Malik. Dependency Modeling in Automated Web Services Quality Component Negotiations. IEEE International Conference on Cybernetics. pp. 110-115, June. (2013).

[14] X Zhao, LW Shen, X Peng \& W Zhao. Finding Preferred Skyline Solutions for SLA-constrained Service Composition. 2013 IEEE 20th International Conference on Web Services. pp. 195-202, June. (2013).

[15] K Ravindran. QoS Auditing for Evaluation of SLA in Cloud-based Distributed Services. 2013 IEEE Ninth World Congress on Services. pp. 257-254, June. (2013).

[16] D.S Pukhkaiev., Kot T.N., Globa L.S., Schill A.. SLA-aware approach for dynamic Web Service Composition. 2013 23rd International Crimean Conference on Microwave and Telecommunication Technology. pp.348-349, Sept. (2013).

[17] D Bruneo, S Distefano, F Longo, M Scarpa. Stochastic Evaluation of QoS in Service-Based Systems. IEEE Transactions on Parallel and Distributed Systems. pp. 2090 - 2099, VOL. 24, NO. 10, OCT. (2013).

[18] C LIjin Zhou Ya . Research on Dynamic Web Service Selection based Multi-QoS constraints . Control and Automation Publication Group.(2009), vol. 25, no. 113, pp. 209-211.

[19] X Zhiyu. Economic Game Theory(3rd).Shanghai: Fudan University Press. (2008).

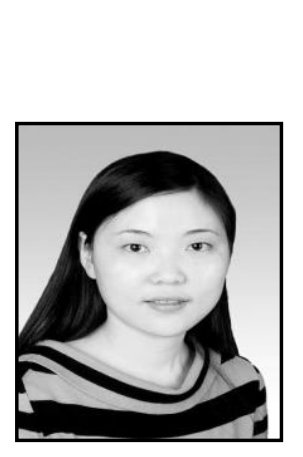

\section{Authors}

Yanping Chen, Professor, Ph.D. Her research interest covers the Service computing,now, she works on School of Computer Science,Xi'an University of Posts and Telecommunications,Xi'an,Shaanxi,China.Email:chenyanping@xup t.edu.cn. 


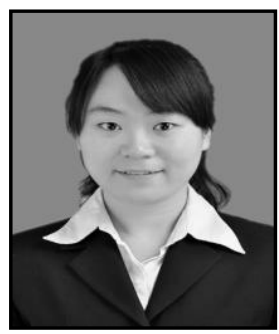

Wei Shi. M.S. Her research interests cover the Web Service and Service-oriented computing. Now she works on Xi'an Jiaotong University City College, Xi'an, Shaanxi, China. Email: sw8864@hotmail.com

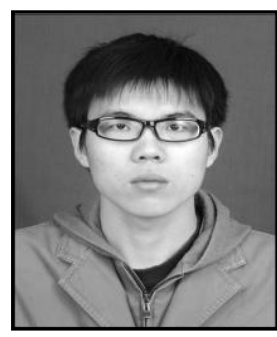

Jue Wang. Ph.D. His research interests cover the Game theory and Numerical Simulation. Now he works on School of Finance and Economics of Xi'an Jiaotong University, Xi'an, Shaanxi, China. Email: wang2jue2@126.com

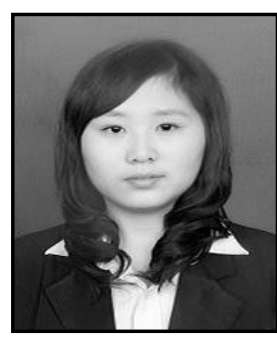

Huifen Yan. Her research interests cover the Web Service .Now she study on School of Computer Science,Xi' an University of Posts and Telecommunications,Xi'an,Shaanxi,China.Email:yanelise@ 163. com

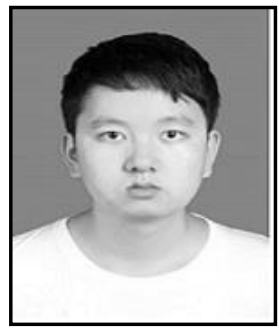

Chao Guo.His research interests cover the Web Service .Now he study on School of Computer Science,Xi' an University of Posts and Telecommunications,Xi'an,Shaanxi,China.

Email:guochao19920408@qq.com 